\title{
Study on Inter-Turn Short Circuit Test for Distribution Reactor
}

\author{
Wei QIN ${ }^{1}$, Tiansong GU, Hongliang LI \\ State Grid Electric Power Research Institute, Wuhan Nari Limited Liability \\ Company, Wuhan 430074, China
}

\begin{abstract}
Inter-turn short circuit is a common fault in reactor. In view of the current situation of insufficient condition based maintenance of reactor, an on-line monitoring method suitable for Inter-turn short circuit fault of distribution reactor was proposed in this paper. Firstly, the equivalent circuit model of reactor was built, and the calculation method of electrical parameters of reactor was obtained; Then, a set of on-line monitoring system for reactor short-circuit fault was built. Finally, the short-circuit test of reactor under different fault location and fault degree was carried out. The results show that the equivalent reactance and resistance change rate of inter-turn short circuit of reactor have the symmetry of short circuit position, the absolute value of change rate in the middle is the largest, and the absolute value of change rate at both ends is the smallest. With the deepening of short-circuit fault, the equivalent reactance decreases and the decreasing range increases. The equivalent resistance shows an increasing trend, and the increasing range increases. This research technology can realize the effective monitoring of inter-turn short circuit fault of distribution reactor.
\end{abstract}

Keywords. Distribution reactor, equivalent reactance, equivalent resistance, position of short circuit, degree of short circuit

\section{Introduction}

With the development of smart grid, the scale of distribution network is becoming larger, the power load is becoming higher, and the harmonic content in distribution network is becoming higher due to large-scale applications such as nonlinear load charging pile. In order to solve this problem, reactors, as inductive equipment, cooperate with capacitors to form passive power filters, which can improve the power quality of power grid [1-2]. Dry-type reactor is widely used in distribution network. Air is the heat sink mode. The short-circuit fault of turn insulation can easily cause the burning of windings [3-5], which may cause serious power accidents.

Electric workers all over the world have carried out a lot of research on the interturn short-circuit monitoring of reactor, which is generally divided into electrical and non-electrical monitoring methods [6-8]. Relevant scholars in colleges and universities [9-11] determine the location of temperature sensor based on the simulation results of temperature field, and monitor the operating status of reactor by measuring the winding temperature. The advantage of this method is simple installation and wide coverage. However, it is not possible to directly measure and calculate the temperature inside the 
reactor. The magnetic induction detection coil method [12-13] uses the principle of electromagnetic induction to detect serious inter-turn short-circuit faults quickly with high sensitivity, but it cannot detect inter-turn short-circuit faults at different positions of the reactor. The zero-sequence amplitude comparison monitoring method [14-15] compares the bus voltage fault component with the compensating voltage fault component after inter-turn faults. Based on the above analysis, this paper presents an online monitoring method for inter-turn short circuit faults of distribution reactors in view of the status of insufficient maintenance of reactor.

\section{Short Circuit Calculation Model}

The original reactor winding is composed of $n$ windings in parallel. Among them, some of the windings of the i-th layer have inter-turn short circuits. The closed loop formed by the short-circuit loop is recorded as the $n+1$ winding. The corresponding equivalent short-circuit model is shown in Figure 1.

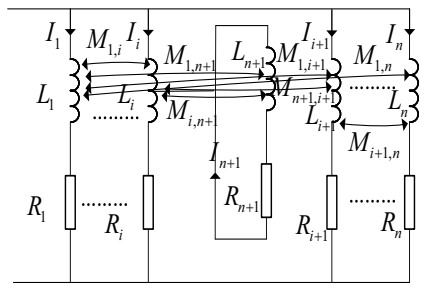

Figure 1. Equivalent short circuit model of reactor.

Second paragraph. Carrying out circuit analysis, each normal parallel winding can be equivalent to the inductance $L_{i}$ and the resistance $R_{i}$ in series, the voltage is equal, denoted as $\mathrm{U}$, and the current is $I_{i}$. Due to the effect of inductance, there is mutual inductance $M_{i, j}$ between the windings. The short-circuit loop voltage is 0 . According to electromagnetic induction, there is still induced current in the short-circuit loop, which is recorded as $I_{n+1}$. After a short-circuit fault occurs, the voltage equations of $n+$ 1 branches are

$\left[\begin{array}{ccccccc}R_{1}+j \omega L_{1} & j \omega M_{1,2} & \ldots & j \omega M_{1, i+1} & \ldots & j \omega M_{1, n} & j \omega M_{1, n+1} \\ \vdots & \vdots & \vdots & \vdots & \vdots & \vdots & \vdots \\ j \omega M_{i, 1} & \cdots & R_{i}+j \omega L_{i} & \cdots & j \omega M_{i, n-1} & j \omega M_{i, n} & j \omega M_{i, n+1} \\ j \omega M_{i+1,1} & j \omega M_{i+1,2} & \ldots & R_{i+1}+j \omega L_{i+1} & \ldots & j \omega M_{i+1, n} & j \omega M_{i+1, n+1} \\ \vdots & \vdots & \vdots & \vdots & \vdots & \vdots & \vdots \\ j \omega M_{n, 1} & j \omega M_{n, 2} & \ldots & j \omega M_{n, i+1} & \ldots & R_{n}+j \omega L_{n} & j \omega M_{n, n+1} \\ j \omega M_{n+1,1} & j \omega M_{n+1,2} & \cdots & j \omega M_{n+1, i+1} & \cdots & j \omega M_{n+1, n} & R_{n+1}+j \omega L_{n+1}\end{array}\right]\left[\begin{array}{c}I_{1} \\ \vdots \\ I_{i} \\ I_{i+1} \\ \vdots \\ I_{n} \\ I_{n+1}\end{array}\right]=\left[\begin{array}{c}U \\ \vdots \\ U \\ U \\ \vdots \\ U \\ 0\end{array}\right]$

\section{Design of Online Monitoring System}

The on-line monitoring system for short-circuit faults of distribution reactor mainly consists of analog and digital circuits. First, the bus-side windings of reactor are collected by high-precision voltage transformer and current transformer. After the signal conditioning module filters, amplifies, removes noise, then outputs the digital signal to 
the control unit ARM through the AD module, completes a series of data processing and data storage work, and finally transmits the reactor voltage and current signals to the data center through wireless communication to achieve real-time monitoring of the reactor failure status, see Figure 2. Among them, the digital circuit can store the data information of the mutual inductor, can analyze in real time, and improves the timeliness of fault identification. At the same time, the data analysis uses a quasi-synchronous algorithm to ensure the accuracy of measurement results.

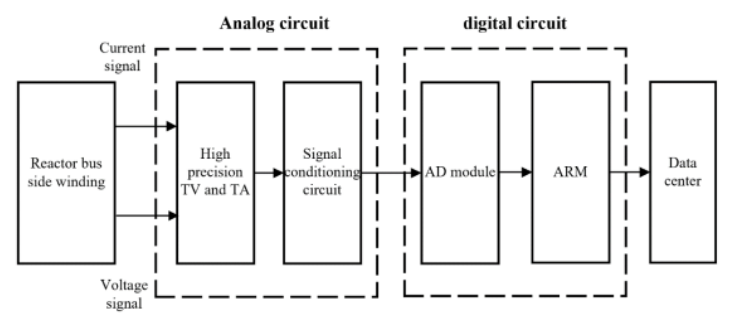

Figure 2. On-line monitoring system.

\subsection{Calculation of equivalent reactance and resistance}

The calculation of the total voltage and current signals of the reactor is shown in the following formulas (2) and (3), respectively.

$$
\begin{aligned}
& u(t)=a_{u d}+\sum_{k=1}^{\infty}\left[a_{u k} \cos \left(2 k \pi f_{0} t\right)+b_{u k} \sin \left(2 k \pi f_{0} t\right)\right] \\
& i(t)=a_{i d}+\sum_{k=1}^{\infty}\left[a_{i k} \cos \left(2 k \pi f_{0} t\right)+b_{i k} \sin \left(2 k \pi f_{0} t\right)\right]
\end{aligned}
$$

In the formula: $f$ is the base frequency; $a_{u d}$, aid are the direct component of $u(t)$, $I(t) ; a_{u k}, a_{i k}$ are the imaginary part of the k-th harmonic component of $u(t), I(t) ; b_{u k}$, $b_{i k}$ are the imaginary part of the $k$-th harmonic component of $u(t), I(t)$.

The real and imaginary parts of the voltage and current fundamental signals are calculated as (4):

$a_{u 1}=\frac{2}{T} \int_{0}^{T} u(t) \cos \left(2 \pi f_{0} t\right) d t$

$b_{u 1}=\frac{2}{T} \int_{0}^{T} u(t) \sin \left(2 \pi f_{0} t\right) d t$

$a_{i 1}=\frac{2}{T} \int_{0}^{T} i(t) \cos \left(2 \pi f_{0} t\right) d t$

$b_{i 1}=\frac{2}{T} \int_{0}^{T} i(t) \sin \left(2 \pi f_{0} t\right) d t$

The amplitude of the base wave signal is calculated as (5)

$$
\begin{aligned}
& u_{m}=\sqrt{a_{u 1}^{2}+b_{u 1}^{2}} \\
& i_{m}=\sqrt{a_{i 1}^{2}+b_{i 1}^{2}}
\end{aligned}
$$

The phase of the fundamental signal is calculated as

$\varphi_{u 1}=\arctan \frac{a_{u 1}}{b_{u 1}}$

$\varphi_{i 1}=\arctan \frac{a_{i 1}}{b_{i 1}}$

Further, the equivalent resistance and reactance values of the reactor at the base wave can be obtained by the following formulas: 


$$
\begin{aligned}
& R=\frac{a_{u 1} a_{i 1}+b_{u 1} b_{i 1}}{a_{i 1}^{2}+b_{i 1}^{2}} \\
& X=\frac{a_{i 1} b_{u 1}-a_{u 1} b_{i 1}}{a_{i 1}^{2}+b_{i 1}^{2}}
\end{aligned}
$$

\subsection{Quasi-synchronization algorithm}

In actual measurement, due to the influence of power quality, the frequency of power network may fluctuate, so the sampling period is not necessarily an integer multiple of the period, which may lead to measurement error, so the same standard is introduced. The core of the quasi-synchronization algorithm is to improve the accuracy of the mean value of periodically sampled signals based on adequate sampling data and appropriate data processing methods.

According to the Fourier expansion, it can be seen that the periodic signal $f(t)$ of voltage or current is decomposed into

$f(t)=a_{0}+\sum_{k=1}^{\infty} a_{k} \sin \left(2 \pi k f_{0} t+\varphi_{k}\right)$

The effective value is calculated as (10)

$\overline{f(t)}=\sqrt{\frac{1}{T} \int_{T_{0}}^{T_{0}+T} f^{2}(t) d t}$

Where: $T$ is the voltage or current signal period; $T_{0}$ is the starting point of integration. Make $x=w t$, Then there

$\overline{f(x)}=\frac{1}{2 \pi} \int_{x_{0}}^{x_{0}+2 \pi} f(x) d x$

Let the number of sampling periods be $\mathrm{N}$ and the period deviation be $\Delta$, The number of sampling points in each cycle is $\mathrm{n}$, in the integral interval $[x 0, x 0+n \times(2 \pi+\Delta)]$ Upper equal interval sampling $n \times N+1$ data, make

$$
F^{1}=\frac{1}{\sum_{i_{0}=0}^{N+i_{0}} \rho_{i_{0}}} \sum_{i_{0}=0}^{N+i_{0}} \rho_{i_{0}} f\left(x_{i_{0}}\right)
$$

The recurrence formula is (12)

$$
F^{n}=\frac{1}{\sum_{i_{0}=0}^{N+i_{0}} \rho_{i_{0}}} \sum_{i_{0}=0}^{N+i_{0}} \rho_{i_{0}} F^{n-1}, n=2.3 \ldots
$$

Among them $\rho_{i_{0}}$ is the weight coefficient, which can be further obtained

$$
\lim _{n \rightarrow \infty} F_{n}=a_{0}=\overline{f(x)}
$$

The sum of the product of the sampling value and the corresponding weight coefficient is the average value.

\section{Data analysis}

In the test, the reactor is dry-type air core type, the voltage level is $35 \mathrm{kV}$, and the equivalent resistance and reactance are $0.182 \Omega$ and $1.24 \Omega$ respectively. 


\subsection{Influence of frequency fluctuation}

Due to the phenomenon of frequency fluctuation in power grid voltage, the whole cycle sampling can not be guaranteed. Considering that under abnormal conditions of power system, the allowable deviation of power supply frequency shall not exceed $\pm 1 \mathrm{~Hz}$ and the frequency fluctuation range is $[49 \mathrm{~Hz} \sim 51 \mathrm{HZ}]$, the equivalent resistance and equivalent reactance of reactors with different frequencies under normal conditions are measured. The calculation formulas of equivalent resistance change rate $d R / d f$ and equivalent reactance change rate $d X / d f$ are shown in equation (14). The values of quasi synchronous algorithm and untreated case are compared and analyzed, as shown in Figure 3 and Figure 4 respectively.

$$
\begin{aligned}
& d R / d f=\frac{\Delta R}{\Delta f}=\frac{R_{1}-R_{2}}{f_{1}-f_{2}} \\
& d X / d f=\frac{\Delta X}{\Delta f}=\frac{X_{1}-X_{2}}{f_{1}-f_{2}}
\end{aligned}
$$

Where: $R_{1}$ and $X_{1}$ are the equivalent resistance value and reactance value of frequency $f_{1}$ respectively; $R_{2}$ and $X_{2}$ are the equivalent resistance value and reactance value of frequency $f_{1}$ respectively; $\Delta R 、 \Delta X 、 \Delta f$ is the change of equivalent resistance, equivalent reactance and frequency respectively.

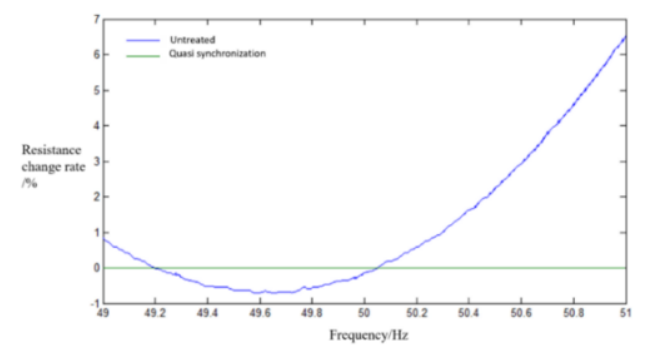

Figure 3. Rate of change for resistance at different frequencies.

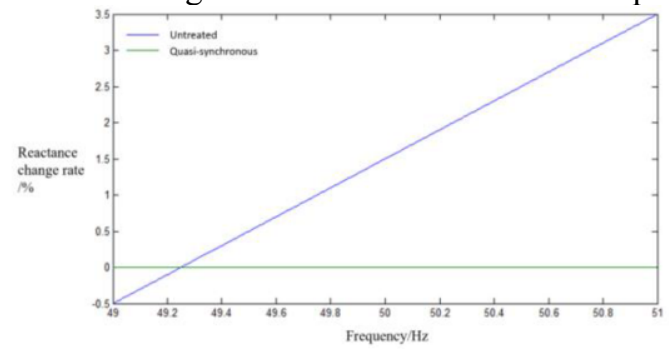

Figure 4. Rate of change for reactor at different frequencies.

As can be seen from Figure 3, the equivalent resistance value changes with the increase of frequency, showing a trend of first decreasing and then increasing. After introducing the quasi synchronous algorithm, the resistance value is almost unchanged. It can be seen from Figure 4 that with the increase of frequency, the equivalent reactance value also gradually increases, showing a positive correlation. After the introduction of quasi synchronous algorithm, the reactance value is almost unchanged. 


\subsection{Effect of temperature rise}

When the reactor works under the rated working condition, the winding temperature will increase and the equivalent reactance and resistance of the reactor will change. In order to further study its stable value, after the voltage, current and other parameters are stable, the equivalent reactance and resistance values will be summarized every $0.5 \mathrm{~h}$. The calculation process of the equivalent resistance change rate $d R / d f$ and the equivalent reactance change rate $d X / d f$ is shown in equation (15)

$d R / d t=\frac{\Delta R}{\Delta t}=\frac{R_{1}-R_{2}}{t_{1}-t_{2}}$
$d X / d t=\frac{\Delta X}{\Delta t}=\frac{X_{1}-X_{2}}{t_{1}-t_{2}}$

Where, $R_{1}$ and $X_{1}$ are the equivalent resistance value and reactance value at time $t_{1}, R_{2}$ and $X_{2}$ are the equivalent resistance value and reactance value at time $t_{2} . \Delta R 、 \Delta X$ and $\Delta t$ are equivalent resistance variation, equivalent reactance variation and time variation respectively. Draw the curve of equivalent resistance change rate and reactance change rate, as shown in Figure 5.

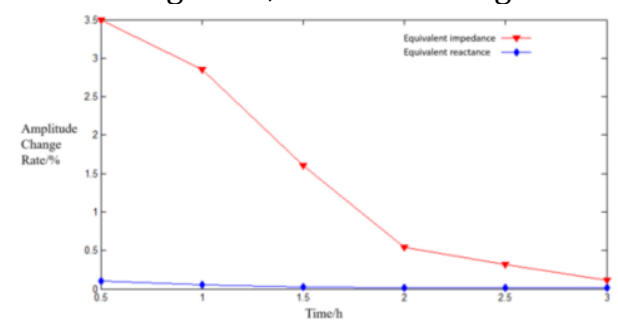

Figure 5. Change rate of equivalent resistance and reactance.

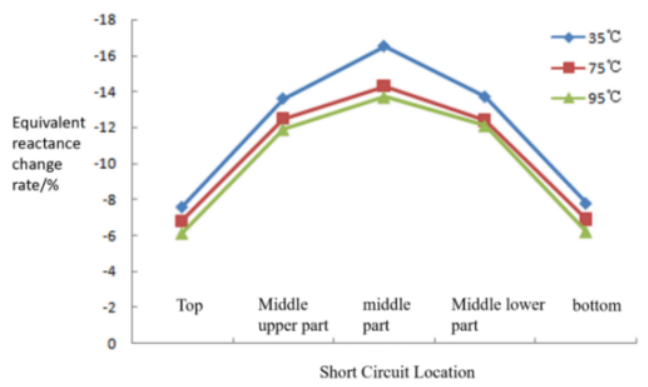

Figure 6. Relationship between the change rate of equivalent reactance and temperature rise.

As can be seen from Fig. 5, with the passage of time, the change rate gradually decreases, the equivalent resistance first increases and then tends to be stable, and the change rate is only $0.5 \%$ after $2 \mathrm{~h}$; Similarly, with the passage of time, the equivalent reactance value increases slightly, and the change rate of equivalent reactance is basically 0 . Therefore, the equivalent reactance and resistance can be measured after $2 \mathrm{~h}$.

The reactor is cylindrical and the winding is wound from inside to outside. According to the axial direction, the winding is divided into five equal parts from 
top to bottom. The short-circuit fault location can be divided into top, middle upper part, middle lower part and bottom, with a total of 5 parts, each accounting for $20 \%$. Introducing inter turn short-circuit simulation leads at different positions of the reactor and manually closing the short-circuit switch can simulate short-circuit faults with different turns at different positions [16-19]. $R^{\prime}$ and $X^{\prime}$ are the equivalent resistance change rate and reactance change rate at the current shortcircuit position respectively. The calculation process is shown in equation (16) $R^{\prime}=\frac{R_{s}-R_{0}}{R_{0}} \times 100 \%$ $X^{\prime}=\frac{X_{s}-X_{0}}{X_{0}} \times 100 \%$

Among them, $R_{0}$ and $X_{0}$ are the equivalent resistance and reactance values under normal conditions, and $R_{S}$ and $X_{S}$ are the equivalent resistance and reactance values under current short circuit position. In order to further study the relationship between equivalent reactance of reactor and resistance and temperature under different turn to turn short circuit positions, common temperatures such as winding temperature $35{ }^{\circ} \mathrm{C}, 75^{\circ} \mathrm{C}$ and $95^{\circ} \mathrm{C}$ are selected for testing, and the curves are drawn as shown in figures 6 and 7 .

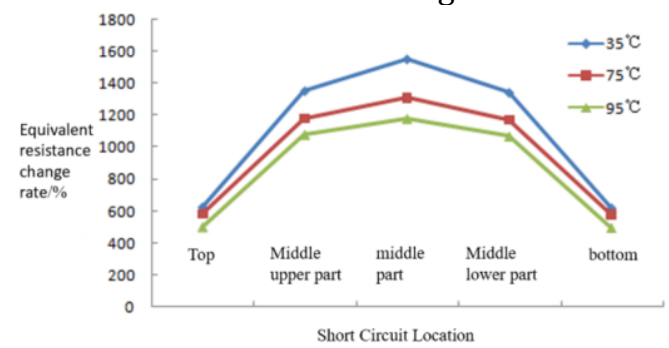

Figure 7. Relationship between the change rate of equivalent resistance and temperature rise.

It can be seen from Fig. 6 that under the symmetrical position, the equivalent reactance rate of inter turn short circuit of reactor is consistent, the absolute value of change rate in the middle is the largest, the absolute value of change rate at both ends is the smallest, and the overall change rate is between $-5.8 \%$ and $-16.2 \%$. Under the same short-circuit position, the absolute value of the change rate of equivalent reactance decreases with the increase of temperature. It can be seen from Figure 7 that the change rate of equivalent resistance of reactor turn to turn short circuit also has the symmetry of short circuit position. The overall change rate is between $4.12 \sim 15.3$ times. At the same short circuit position, the change rate of equivalent resistance decreases with the increase of temperature.

\section{3. influence of different short circuit degree}

Define fault degree $s t$ as the ratio of short-circuit winding height $h_{s t}$ to total reactor winding height $h_{t}$, i.e

$s t=\frac{h_{s t}}{h_{t}} \times 100 \%$

Obviously, according to the short circuit position, there is $s t \leq 20 \%$, for further quantification, it is divided into three intervals [0\% 7\%), [7\% 14\%) and 
[14\% 20\%], corresponding to mild fault, moderate fault and severe fault respectively.

The curves of equivalent reactance and equivalent resistance with shortcircuit position under different fault degrees are drawn, as shown in Fig. 8 and Fig. 9 respectively, in which mild fault, moderate fault and severe fault are $6 \%, 12 \%$ and $18 \%$ respectively.

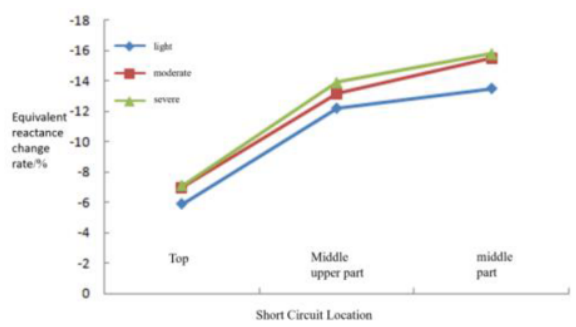

Figure 8. Relation between degree of short circuit and change rate of reactance.

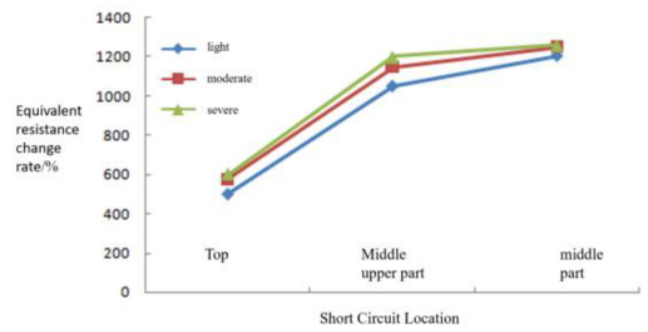

Figure 9. Relation between degree of short circuit and change rate of resistance.

The winding temperature is $95^{\circ} \mathrm{C}$. Due to the symmetry of the short circuit position, only the top, middle, upper and middle short circuits are studied here. As can be seen from Fig. 8, with the deepening of short-circuit fault, the equivalent reactance decreases, and the decreasing range increases. Similarly, it can be seen from Figure 9 that with the deepening of short-circuit fault, the equivalent resistance tends to increase, and the increasing range increases.

\section{Conclusion}

In this paper, a set of on-line monitoring system for reactor short-circuit fault is built, and the influencing factors of equivalent reactance and resistance are studied. The main conclusions are as follows:1) The equivalent reactance and resistance change rate of inter turn short circuit of reactor have the symmetry of short circuit position. The absolute value of change rate in the middle is the largest and the absolute value of change rate at both ends is the smallest. Under the same shortcircuit position, the absolute values of the change rate of equivalent reactance and equivalent resistance decrease with the increase of temperature.2) With the deepening of short-circuit fault, the equivalent reactance decreases and the decreasing range increases; The equivalent resistance shows an increasing trend, and the increasing range increases. 


\section{References}

[1] Zhang L, Jia-Sheng L, Wang Y H. Field test on the turn-to-turn insulation for 35k V dry-type aircore reac-tors[J]. Electric Machines \& Control, 2014, 18(06): 66-71.

[2] Wang Ping, Zhao Yingyu, Lv Fangcheng et al. Effect of rain dis-tribution on surface electric field of \pm 1 $100 \mathrm{kv}$ smoothing reactor de-sign[J]. High Voltage Engineering, 2017(10):75-81.

[3] Gao xiaodong, Qu Wentao, Chen Rengang. Fault analysis and pre-ventive measures on 35 kv air-core drytype reactor[J]. Power Ca-pacitor \& Reactive Power Compensation, 2015, 36(2): 85-88.

[4] Fu Weiping, Zhao Jingwu, Huo Chunyan.Reason Analysis for a 35kV Dry Type Reactor Fault[J]. Power Capacitor \& Reactive Power Compensation, 2011, 32(01): 59-62.

[5] Zhang Bo. Application of Reflux Reactor in Solving Problem of In-sulation Burning at Station Rail End[J]. Railway Signalling \& Communication Engineering, 2019, 16(S1): 16-19.

[6] $\mathrm{Wu}$ Dongwen. Temperature field distribution and infrared tempera-ture measurement method research of $35 \mathrm{kv}$ dry-type reactor[J]. Transformer, 2013, 50(9): 62-65.

[7] PAN Zhen, JI Donghong, ZHANG Wenqing, et al. Explosion analysis on $330 \mathrm{kV}$ current transformer [J]. Power Capacitor\& Reactive Pow-er Compensation, 2017, 38(1): 90-92.

[8] JIANG Zhipeng, WEN Xishan, WANG Yu, et al. Test and coupling calculation of temperature field for uhv dry-type air-core smoothing reactor[J]. Proceedings of the CSEE, 2015, 35(20): 5344-5350.

[9] Zhang Jie,Tan Xiangyu,Wang Ke et al. Application of distributed fi-ber bragg grating sensor system in dry-type air-core reactor[J]. Sci-ence Technology and Engineering, 2015(16): 160-164.

[10] Sun Zhiyong. Design of on-line monitoring and fire warning system of dry-type reactor[D]. ShangDong: Shangdong University, 2016: 16-18.

[11] Jiang Zhipeng, Wen Xishan,Wang Yu,et al.Test and Coupling Calcu-lation of Temperature Field for UHV Dry-Type Air-Core Smoothing Reactor[J]. Proceedings of the CSEE, 2015, 35(20): 5344-5350. [23]

[12] LI Ying, YANG Songwei, ZHANG Jianhuan, et al. Study of temperature monitoring method of dry-type air-core reactor based on distributed optical fiber[J]. Instrument Technique and Sensor, 2017(2): 43-46,50.

[13] WANG Xiaowen, LIU Yubo. The study based on detecting coil for dry-type shunt reactors fault detection[J]. Shenyang Institute of Engineering (Natural Science), 2016, 12(4): 336-339.

[14] LI Xiaoxiao,YU Xiangyang. Characteristic analysis of three-phase four-leg inverter based load unbalance compensator for mi-crogrid[J]. Power System and Clean Energy, 2017,33(5): 13-17.

[15] Lin Shenghong. Research on the Protection between turns of high voltage shunt reactors[D]. Nanjing:Nanjing Normal University, 2016.

[16] Huang Xinbo,Zhang Long,Zhu Yongchan, et al. Online inter-turn in-sulation monitoring technology based on power angle characteristics for dry-type air-core reactor[J]. Electric Power Automation Equipment, 2019, 39(02): 143-148.

[17] LIU Chuanghua,LIU Mei. Detection technology of the interturn insulation of dry-type air-core reactor J]. Proceedings of the CSUEPSA, 2016, 28 (Supplement): 115-118.

[18] GUO Xiangfu, MENG Bo. Interturn short circuit fault current and overcurrent protective system of shunt reactor with air core[J]. Transformer, 2008, 45(11): 20-22.

[19] ZHANG Bin, HUANG Wenwu, LING Yun, et al. Simulation on transient characteristics of inter-turn short circuit fault of dry-type aircore reactor based on ANSYS Maxwell[C] // International Conference on Electrical Engineering and Automation Con-trol (ICEEAC2017). Nanjing,China:[s. n.],2017: 416422. 